\title{
Designer Molecules for Fundamental-Symmetry Tests
}

\author{
Researchers design radioactive molecules that might have exceptional \\ sensitivity to the symmetry violations explaining the matter-antimatter \\ imbalance of the Universe.
}

By Ronald F. Garcia Ruiz

$$
\text { I }
$$

$f$ the laws of physics were perfectly symmetric, matter and antimatter should have been created in equal amounts after the big bang. The visible Universe, however, is overwhelmingly comprised of matter, with antimatter making up just a tiny fraction-about one part per billion. Explaining this observed matter-antimatter imbalance is one of the major open questions in physics. Particle physicists have run many experiments to search for an answer, revealing subtle violations of certain fundamental symmetries. The asymmetries observed so far, however, are too small to explain the cosmic imbalance. Two independent teams now report important steps in a

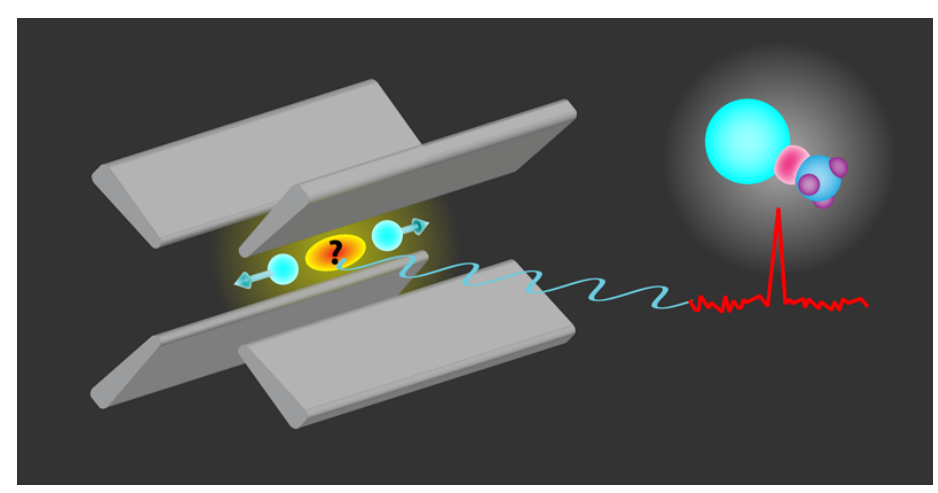

Figure 1: Researchers have produced molecules through chemical reactions between trapped, laser-cooled radium ions and molecules of methanol [1]. The radium ions and a few of the molecules arrange in an ordered structure called a Coulomb crystal. Through a mass spectrometry technique, the researchers have identified the produced molecular species, demonstrating that they include molecules containing radium.

Credit: Max Ladabaum/UCSB; adapted by APS/Alan Stonebraker promising approach for testing fundamental symmetry violations through their effects on the spectra of polar, radioactive molecules. Mingyu Fan of the University of California, Santa Barbara, and co-workers have experimentally demonstrated a laser-based method for creating and identifying molecules containing isotopes of radium [1]. Phelan $\mathrm{Yu}$ and Nicholas Hutzler, both at the California Institute of Technology, have performed a detailed theoretical study of these molecules, revealing that they are extraordinarily sensitive probes of fundamental symmetries [2].

A necessary condition for generating matter-antimatter asymmetry is the violation of charge conjugation-parity symmetry, or $C P$ symmetry. This symmetry implies that the laws of physics are the same if a particle is replaced with the corresponding antiparticle while the particle spatial coordinates are inverted. Some level of $C P$ violation is predicted by the standard model of particle physics and was experimentally observed in certain decays of $B$ or $D$ mesons. More recently, experiments also suggested $C P$ violation in neutrino oscillations [3]. But stronger violations are needed to explain the Universe's imbalance, so physicists across different fields have dedicated intense efforts to predicting and testing new sources of $C P$ violation (or of its equivalent time-reversal-symmetry violation).

Theoretical studies suggest that molecules containing one or more radioactive nuclei can offer unprecedented sensitivity to symmetry violations [4-6]. CP violating forces in the molecules' nuclei can produce a collective electric dipole moment (EDM) that in turn induces a charge displacement in atoms and molecules-quantified by the so-called Schiff moment [7], 
which can induce measurable shifts in energy levels of the molecule. This effect is theorized to scale with atomic mass and to be dramatically enhanced in nuclei with octupole deformation such as radium-225, which has atomic number $\mathrm{Z}=88$ and a pear-shaped nucleus [8]. Molecules containing these isotopes, like $\mathrm{RaOH}^{+}$, are promising candidates for spotting these Schiff-moment effects [5, 6]. However, nuclei like radium-225 are short lived and can only be produced in small quantities. As a result of these challenges, experiments on molecules containing these rare nuclei are scarce [9].

Fan and colleagues have demonstrated a new method for creating and identifying radioactive molecules. Their scheme (Fig. 1) involves using dynamic electric fields to trap laser-cooled $\mathrm{Ra}^{+}$ions, which are then mixed with methanol vapor. This setup facilitates a chemical reaction that produces $\mathrm{RaOH}^{+}$or $\mathrm{RaOCH}_{3}^{+}$molecules. A few of these charged molecules, together with the laser-cooled $\mathrm{Ra}^{+}$ions, form an ordered structure called a Coulomb crystal. Identifying these new molecular compounds, however, isn't easy. Since their transition energies are unknown and are expected to be different from those of the isolated $\mathrm{Ra}^{+}$, the molecules cannot yet be directly identified by their characteristic spectral signatures.

To overcome this identification challenge, the researchers developed an all-optical nondestructive method that measures the frequency with which the Coulomb crystal oscillates around its equilibrium position in the ion trap. In their method, fluorescent light from the $\mathrm{Ra}^{+}$ions is collected by a photodetector placed at a fixed position. Thus, the motion of the ions causes temporal variations of the observed light intensity, with well-defined peaks in the frequency spectrum of the signal. As the motion of the ions is affected by the mass of the co-trapped species, the mass of the synthesized molecules can be deduced from changes in the oscillation frequencies. In their first demonstration of the method-which they made using the long-lived radium-226 isotope-Fan and colleagues measured, in only three seconds, the mass of the molecular species with a fractional uncertainty of one part in eight hundred.

Yu and Hutzler undertook a detailed theoretical investigation of the newly observed $\mathrm{RaOCH}_{3}^{+}$molecule, assuming that the molecule's radium is the isotope with atomic mass 225 . Using $a b$ initio calculations, the researchers computed the molecule's electronic structure, focusing on those hyperfine states that should be particularly sensitive to $C P$ violations. Their predictions showed that $\mathrm{RaOCH}_{3}^{+}$offers unique advantages for Schiff-moment measurements, in particular because it is highly polarizable with small external electric fields. This molecule has another important asset for high-precision studies: Its internal field can be reversed by switching its polarization. By comparing measurements made under opposite electric fields, experimenters can mitigate systematic uncertainties [10]. Yu and Hutzler show that the extreme sensitivity to symmetry violations could lead to observable shifts in the molecular energy levels even in experiments with a single trapped molecule.

These combined experimental and theoretical developments represent exciting progress in this rapidly moving field of research and are critical to spur future work. Significant experimental challenges will have to be overcome to extend the method developed by Fan and colleagues, so that it can be applied to produce molecules containing radium-225 and to precisely characterize their structure. The payoff, as revealed by the theoretical work of Yu and Hutzler, would be a promising platform for uncovering beyond-standard-model physics, which might be sensitive not only to symmetry violations but also to new scalar fields and dark matter. But exciting applications can be envisioned in a diverse range of fields, as radioactive molecules could allow researchers to probe phenomena that wouldn't be accessible with stable molecules. "Designer" radioactive molecules could, for instance, enable systematic studies of electron-nucleus interaction in molecules containing nuclei with extreme numbers of protons or neutrons. And knowledge of molecular spectra could be the basis of radioisotopic dating techniques applicable to astrophysics [11].

Ronald F. Garcia Ruiz: Department of Physics, Massachusetts Institute of Technology, Cambridge, MA, USA

\section{REFERENCES}

1. M. Fan et al., "Optical mass spectrometry of cold $\mathrm{RaOH}^{+}$and $\mathrm{RaOCH}_{3}^{+}$," Phys. Rev. Lett. 126, 023002 (2021).

2. P. Yu and N. R. Hutzler, "Probing fundamental symmetries of deformed nuclei in symmetric top molecules," Phys. Rev. Lett. 126, 023003 (2021).

3. K. Abe et al. (The T2K Collaboration), "Constraint on the 
matter-antimatter symmetry-violating phase in neutrino oscillations," Nature 580, 339 (2020).

4. T. A. Isaev et al., "Laser-cooled RaF as a promising candidate to measure molecular parity violation," Phys. Rev. A 82, 052521 (2010).

5. T. A. Isaev et al., "Laser-coolable polyatomic molecules with heavy nuclei,” J. Phys. B 50, 225101 (2017).

6. I. Kozyryev and N. R. Hutzler, "Precision measurement of time-reversal symmetry violation with laser-cooled polyatomic molecules," Phys. Rev. Lett. 119, 133002 (2017).

7. T. E. Chupp et al., "Electric dipole moments of atoms, molecules, nuclei, and particles," Rev. Mod. Phys. 91, 015001
(2019).

8. L. P. Gaffney et al., "Studies of pear-shaped nuclei using accelerated radioactive beams," Nature 497, 199 (2013).

9. R. F. Garcia Ruiz et al., "Spectroscopy of short-lived radioactive molecules," Nature 581, 396 (2020).

10. W. B. Cairncross et al., "Precision measurement of the electron's electric dipole moment using trapped molecular ions," Phys. Rev. Lett. 119, 153001 (2017).

11. T. Kamiński et al., "Astronomical detection of radioactive molecule ${ }^{26} \mathrm{AlF}$ in the remnant of an ancient explosion," Nat. Astron. 2, 778 (2018). 